\title{
Complexity of Simple Dependent Bimodal Logics
}

\author{
Stéphane Demri \\ Laboratoire LEIBNIZ-CNRS, U.M.R. 5522 \\ 46 Avenue Felix Viallet, 38031 Grenoble, France \\ Email: demri@imag.fr
}

\begin{abstract}
We characterize the computational complexity of simple dependent bimodal logics. We define an operator $\oplus \subseteq$ between logics that almost behaves as the standard joint operator $\oplus$ except that the inclusion axiom [2]p $\Rightarrow[1] \mathrm{p}$ is added. Many multimodal logics from the literature are of this form or contain such fragments. For the standard modal logics $K, T, B, S 4$ and $S 5$ we study the complexity of the satisfiability problem of the joint in the sense of $\oplus$ c. We mainly establish the PSPACE upper bounds by designing tableaux-based algorithms in which a particular attention is given to the formalization of termination and to the design of a uniform framework. Reductions into the packed guarded fragment with only two variables introduced by M. Marx are also used. E. Spaan proved that $K \oplus \subset S 5$ is EXPTIME-hard. We show that for $\left\langle\mathcal{L}_{1}, \mathcal{L}_{2}\right\rangle \in\{K, T, B\} \times\{S 4, \bar{S} 5\}, \mathcal{L}_{1} \oplus \subseteq \mathcal{L}_{2}$ is also EXPTIME-hard.
\end{abstract}

\section{Introduction}

Combining logics The combination of modal logics has deserved in the past years a lot of attention (see e.g. $[12,15,21,16,2,24]$ ) and this is an exciting area. Indeed, not only there are many ways to combine logics (fusion, product, ...) but also many properties of the combined logics deserve to be studied (completeness, compactness, finite model property, interpolation, decidability, complexity, ... ). In this paper, we are mainly concerned with computational complexity issues and as a side-effect with the design of tableaux-based decision procedures. The simplest way to combine two logics is to take their fusion, that is to obtain a bimodal logic which has no axioms that use both of the operators. For two normal modal logics $\mathcal{L}_{1}$ and $\mathcal{L}_{2}$, we write $\mathcal{L}_{1} \oplus \mathcal{L}_{2}$ to denote the smallest bimodal logic with two independent modal operators, say [1] and [2]. The complexity of such logics has been analyzed in [19] and from [22,18], we know that for instance the logics $\mathrm{K} \oplus \mathrm{K}, \mathrm{S} 5 \oplus \mathrm{S} 5, \mathrm{~S} 4 \oplus \mathrm{S} 5$ and $\mathrm{K} \oplus \mathrm{S} 5$ have PSPACE-complete satisfiability problems.

Other combinators for modal logics are relevant (see e.g. $[15,16]$ ). We write $\mathcal{L}_{1} \oplus \subset \mathcal{L}_{2}$ to denote the smallest bimodal logic containing $\mathcal{L}_{1} \oplus \mathcal{L}_{2}$ and the axiom schema $[2] \mathrm{p} \Rightarrow[1] \mathrm{p}$. It is not very difficult to design new operators since each recursive set of bimodal formulae potentially induces a way to combine two logics. The fusion operator $\oplus$ is simply associated to the empty set of formulae. In the paper, we investigate the complexity of bimodal logics obtained from monomodal 
logics by application of $\oplus \subseteq$. To be more precise, we adopt a semantics-oriented definition since we define an operator $\oplus c$ on classes of monomodal frames. The logics of the form $\mathcal{L}_{1} \oplus \subseteq \mathcal{L}_{2}$ are said to be simple dependent bimodal logics. For instance, adding a universal modal operator to certain monomodal logics corresponds exactly to operating with $\oplus \subset$. Unlike $\oplus, \oplus \subset$ does not preserve decidability since by [30], a Horn modal logic whose satisfiability is in NP, is shown to be undecidable when extended with the universal modal operator. Complexity neither transfers. Indeed, $K \oplus \subset S 5$ has an EXPTIME-hard satisfiability problem [30] although $K$-satisfiability is PSPACE-complete and S5-satisfiability is NP-complete [22]. In this paper, we analyze the complexity of the logics $\mathcal{L}_{1} \oplus \subseteq \mathcal{L}_{2}$ for $\mathcal{L}_{1}, \mathcal{L}_{2} \in\{K, T, B, S 4, S 5\}$. To establish the PSPACE upper bounds, we design Ladner-like algorithms [22, 18,31] (see also [20,26,35] for proof-theoretical analyses) that are known to be close to tableau-based procedures. We invite the reader to consult [8] for understanding how the semantical analysis in [22] can be given a proof-theoretical interpretation. Furthermore, the (semantical) analysis developed in the paper can be plug into a labelled tableaux calculus for the logics. Actually, such a calculus is not difficult to define for such logics following for instance [1].

One may wonder why the operator $\oplus \subset$ c deserves some interest. After all, any bimodal formula generates an operator on logics. Actually, many logics can be explained in terms of $\oplus \subseteq$. Below are few examples:

- the propositional linear temporal logic PLTL with future $F$ and next $X$ : PLTL-satisfiability is PSPACE-complete whereas the fragment with $F$ only [resp. with $X$ only] is in NP [29];

- the logic $\mathrm{S} 4+5$ is shown to have a satisfiability problem equal to the satisfiability problem for $S 5 \oplus \subseteq S 4$ [33];

- many other logics have valid formulae of the form $[2] \mathrm{p} \Rightarrow[1] \mathrm{p}$, from epistemic logics to provability bimodal logics (see e.g. [36]) passing via variants of dynamic logic approximating the Kleene star operator [7];

- information logics derived from information systems (see e.g. [34]).

Our contribution. The technical contribution of the paper is to characterize the computational complexity of the satisfiability problem for the logics $\mathcal{L}_{1} \oplus \subset \mathcal{L}_{2}$ with $\mathcal{L}_{1}, \mathcal{L}_{2} \in\{K, T, B, S 4, S 5\}$ (see e.g. [27] for a thourough introduction to complexity theory). The choice of the logics is a bit arbitrary since many other standard modal logics would deserve such an analysis (the standard modal logics D, K4, G, S4.3, S4.3.1 to quote a few of them). However, we felt that with the present sample, we could reasonably show the peculiarity of $\oplus c$ and how the Ladner-like algorithms are precious to establish PSPACE upper bounds. Moreover, many proofs can be adapted to other logics not explicitly studied here. By way of example, $D \oplus \subseteq K 4$ can be shown to be EXPTIME-complete. In Table 1, we summarize the results. In the table, each problem in a given class 
is complete for the class with respect to logarithmic space transformations ${ }^{1}$. A generalization from the bimodal case to the case with $n \geq 2$ modal connectives is sketched in Section 6 and is planned to be fully treated in a longer version.

\begin{tabular}{|c|c|c|c|c|c|}
\hline$\oplus \mathrm{C}$ & $\mathrm{K}$ & $\mathrm{T}$ & $\mathrm{B}$ & $\mathrm{S} 4$ & $\mathrm{~S} 5$ \\
\hline $\mathrm{K}$ & PSPACE & PSPACE & PSPACE & EXPTIME & EXPTIME \\
\hline $\mathrm{T}$ & PSPACE & PSPACE & PSPACE & EXPTIME & EXPTIME \\
\hline $\mathrm{B}$ & PSPACE & PSPACE & PSPACE & EXPTIME & EXPTIME \\
\hline S4 & PSPACE & PSPACE & PSPACE & PSPACE & PSPACE \\
\hline S5 & PSPACE & PSPACE & PSPACE & PSPACE & NP \\
\hline \hline
\end{tabular}

Table 1. Worst-case complexity of simple dependent bimodal logics

The second contribution consists in comparing the operators $\oplus$ and $\oplus c$. Although we know that $\oplus$ preserves for instance decidability (see e.g. [21]), $\oplus \subset$ does not, even if we restrict ourselves to logics with NP-complete satisfiability problems (see e.g. [30]). It is known that for $\mathcal{L}_{1}, \mathcal{L}_{2} \in\{K, T, B, S 4, S 5\}$, $\mathcal{L}_{1} \oplus \mathcal{L}_{2}$ has a PSPACE-complete satisfiability problem. By contrast, we show that for $\left\langle\mathcal{L}_{1}, \mathcal{L}_{2}\right\rangle \in\{K, T, B\} \times\{S 4, S 5\}, \mathcal{L}_{1} \oplus \subseteq \mathcal{L}_{2}$ is EXPTIME-complete. In a sense, the EXPTIME-hardness of the minimal normal modal logic $\mathrm{K}$ augmented with the universal modal connective, should not necessarily be explained by the presence of the universal modal connective but rather as due to the dependent combinaison of a logic between $\mathrm{K}$ and $\mathrm{B}$ and a logic between $\mathrm{K} 4$ and S5. This reinterpretation is another original aspect of our investigation.

The last but not least contribution stems in the design of parametrized Ladner-like algorithms that allows to establish PSPACE upper bounds. We shall come back to this point throughout the paper.

\section{Bimodal logics}

For any set $X$, we write $X^{*}$ to denote the set of finite strings built from elements of $X . \lambda$ denotes the empty string. For any finite string $s$, we write $|s|$ [resp. last $(s)$ ] to denote its length [resp. the last element of $s$, if any]. For $s \in X^{*}$, for $j \in\{1, \ldots,|s|\}$, we write $s(j)$ [resp. $s[j]]$ to denote the $j$ th element of $s$ [resp. to denote the initial substring of $s$ of length $j]$. By convention $s[0]=\lambda$. For any $s \in X^{*}$, we write $s^{k}$ to denote the string composed of $k$ copies of $s$. For instance, $(1.2)^{2}=1.2 .1 .2$ and $\left|(1.2)^{2}\right|=4$.

Given a countably infinite set For $_{0}=\left\{\mathrm{p}_{0}, \mathrm{p}_{1}, \mathrm{p}_{2}, \ldots\right\}$ of propositional variables the bimodal formulae $\phi$ are inductively defined as follows: $\phi::=\mathrm{p}_{k}$ |

\footnotetext{
${ }^{1}$ In Table 1, we consider the satisfiability problem for $\mathcal{L}_{1} \oplus \subseteq \mathcal{L}_{2}$ (or equivalently the consistency problem). In order to get the table for the validity problem (or equivalently the theoremhood problem), replace NP by coNP.
} 
$\phi_{1} \wedge \phi_{2}|\neg \phi \quad|[1] \phi \mid[2] \phi$ for $\mathrm{p}_{k} \in$ For $_{0}$. The monomodal formulae are bimodal formulae without occurrences of [2]. We write $|\phi|$ to denote the length of the formula $\phi$, that is the length of the string $\phi$. We write $m d(\phi)$ to denote the modal degree of $\phi$, that is the modal depth of $\phi$. md is naturally extended to finite sets of formulae, understood as conjunctions and by convention $m d(\emptyset)=0$. For $j \in\{1,2\},[j]^{i} \phi$ is inductively defined as follows: $[j]^{0} \phi=\phi$ and $[j]^{i+1} \phi=[j][j]^{i} \phi$ for $i \geq 0$. For $s \in\{[1],[2]\}^{*}$, an $s$-formula is defined as a formula prefixed by $s$.

A monomodal [resp. bimodal] frame $\mathcal{F}$ is a structure of the form $\left\langle W, R_{1}\right\rangle$ [resp. $\left.\left\langle W, R_{1}, R_{2}\right\rangle\right]$ such that $W$ is a non-empty set, $R_{1}$ is a binary relation on $W$ [resp. $R_{1}$ and $R_{2}$ are binary relations on $W$ ]. A monomodal [resp. bimodal] model $\mathcal{M}$ is a structure of the form $\left\langle W, R_{1}, m\right\rangle$ [resp. $\left.\left\langle W, R_{1}, R_{2}, m\right\rangle\right]$ such that $\left\langle W, R_{1}\right\rangle$ [resp. $\left.\left\langle W, R_{1}, R_{2}\right\rangle\right]$ is a monomodal [resp. bimodal] frame and $m$ is a map $m:$ For $_{0} \rightarrow \mathcal{P}(W)$. $\mathcal{M}$ is said to be based on the frame $\left\langle W, R_{1}\right\rangle$ [resp. $\left.\left\langle W, R_{1}, R_{2}\right\rangle\right]$. Most of the time we omit the terms 'monomodal' and 'bimodal' when it is clear from the context what kind of objects we are dealing with.

As is usual, the formula $\phi$ is satisfied by the world $w \in W$ in $\mathcal{M} \stackrel{\text { def }}{\Leftrightarrow} \mathcal{M}, w \models \phi$ where the satisfaction relation $\models$ is inductively defined as follows:

$-\mathcal{M}, w \models \mathrm{p} \stackrel{\text { def }}{\Leftrightarrow} w \in m(\mathrm{p})$, for every propositional variable $\mathrm{p}$;

$-\mathcal{M}, w \models[1] \phi \stackrel{\text { def }}{\Rightarrow}$ for every $w^{\prime} \in R_{1}(w), \mathcal{M}, w^{\prime} \models \phi$;

$-\mathcal{M}, w \models[2] \phi \stackrel{\text { def }}{\Leftrightarrow}$ for every $w^{\prime} \in R_{2}(w), \mathcal{M}, w^{\prime} \models \phi$ when $\mathcal{M}$ is bimodal.

We omit the standard conditions for the propositional connectives. Let $\mathcal{C}$ be a class of monomodal [resp. bimodal] frames. A monomodal [resp. bimodal] formula is said to be $\mathcal{C}$-satisfiable $\stackrel{\text { gef }}{g}$ there is a model $\mathcal{M}$ based a frame $\mathcal{F} \in \mathcal{C}$ such that $\mathcal{M}, w \models \phi$ for some $w \in W$. We write $S A T(\mathcal{C})$ to denote the class of $\mathcal{C}$-satisfiable formulae. We write $\mathcal{F} \models \phi$ to denote that for any model $\mathcal{M}$ based on $\mathcal{F}$ and for $w$ in $\mathcal{M}, \mathcal{M}, w \models \phi$. The following standard classes of monomodal frames shall be used:

$-\mathcal{C}_{K}$ is the class of all the frames; $\mathcal{C}_{K}^{\prime}$ [resp. $\left.\mathcal{C}_{T}, \mathcal{C}_{K 4}\right]$ is the class of frames $\left\langle W, R_{1}\right\rangle$ such that $R_{1}$ is irreflexive [resp. reflexive, transitive];

$-\mathcal{C}_{B}$ [resp. $\left.\mathcal{C}_{S 4}\right]$ is the class of frames $\left\langle W, R_{1}\right\rangle$ such that $R_{1}$ is reflexive and symmetric [resp. and transitive];

$-\mathcal{C}_{S 5}\left[\right.$ resp. $\left.\mathcal{C}_{S 5}^{\prime}\right]$ is the class of frames $\left\langle W, R_{1}\right\rangle$ such that $R_{1}$ is an equivalence relation [resp. $\left.R_{1}=W \times W\right]$.

It is known that $S A T\left(\mathcal{C}_{K}\right)=S A T\left(\mathcal{C}_{K}^{\prime}\right)$ and $S A T\left(\mathcal{C}_{S 5}\right)=S A T\left(\mathcal{C}_{S 5}^{\prime}\right)$. Let $\mathcal{C}_{i}$ for $i=1,2$ be classes of monomodal frames. We write $\mathcal{C}_{1} \oplus \mathcal{C}_{2}$ [resp. $\left.\mathcal{C}_{1} \oplus \subset \mathcal{C}_{2}\right]$ to denote the class of bimodal frames $\left\langle W, R_{1}, R_{2}\right\rangle$ such that $\left\langle W, R_{i}\right\rangle \in \overline{\mathcal{C}_{i}}$ for $i=1,2$ [resp. and $R_{1} \subseteq R_{2}$ ].

A normal monomodal [resp. bimodal] logic is a set $\mathcal{L}$ of monomodal [resp. bimodal] formulae such that: $\mathcal{L}$ contains all propositional tautologies, $\mathcal{L}$ is closed under substitution, $[j](\mathrm{p} \Rightarrow \mathrm{q}) \Rightarrow([j] \mathrm{p} \Rightarrow[j] \mathrm{q})$ for $j \in\{1\}$ [resp. for $j \in\{1,2\}]$, $\mathcal{L}$ is closed under modus ponens and $\mathcal{L}$ is closed under generalization, i.e. if $\phi \in \mathcal{L}$, then $[j] \phi$ for $j \in\{1\}$ [resp. for $j \in\{1,2\}]$. Let $K$ be the minimal normal monomodal logic. Below are other standard normal modal logics: $T \stackrel{\text { def }}{=}$ 
$K+[1] \mathrm{p} \Rightarrow \mathrm{p}, B \stackrel{\text { def }}{=} T+\mathrm{p} \Rightarrow[1] \neg[1] \neg \mathrm{p}, S 4 \stackrel{\text { def }}{=} T+[1] \mathrm{p} \Rightarrow[1][1] \mathrm{p}$, $S 5 \stackrel{\text { def }}{=} S 4+\mathrm{p} \Rightarrow[1] \neg[1] \neg \mathrm{p}$. A monomodal [resp. bimodal] logic $\mathcal{L}$ is said to be complete with respect to the class $\mathcal{C}$ of monomodal [resp. bimodal] frames $\stackrel{\text { def }}{\Leftrightarrow}$ for any monomodal [resp. bimodal] formula $\phi, \phi \in S A T(\mathcal{C})$ iff $\neg \phi \notin \mathcal{L}$. For any modal $\operatorname{logic} \mathcal{L}$, we write $\mathcal{C}_{\mathcal{L}}$ to denote the class of frames $\mathcal{F}$ such that for $\phi \in \mathcal{L}$, $\mathcal{F} \models \phi$. This notation $\mathcal{C}_{\mathcal{L}}$ is consistent with the classes of frames defined above.

Here is a first important difference between $\oplus$ and $\oplus \subset$. Corollary 3.1.3 in [30] states that if $\mathcal{C}_{1}, \mathcal{C}_{1}^{\prime}, \mathcal{C}_{2}, \mathcal{C}_{2}^{\prime}$ are classes of monomodal frames closed under disjoint unions and if $S A T\left(\mathcal{C}_{1}\right)=S A T\left(\mathcal{C}_{1}^{\prime}\right)$ and $S A T\left(\mathcal{C}_{2}\right)=S A T\left(\mathcal{C}_{2}^{\prime}\right)$, then $S A T\left(\mathcal{C}_{1} \oplus \mathcal{C}_{2}\right)=S A T\left(\mathcal{C}_{1}^{\prime} \oplus \mathcal{C}_{2}^{\prime}\right)$. By constrast, $S A T\left(\mathcal{C}_{T} \oplus \subseteq \mathcal{C}_{K}^{\prime}\right) \neq S A T\left(\mathcal{C}_{T} \oplus \subseteq \mathcal{C}_{K}\right):$ $\mathcal{C}_{T} \oplus \subset \mathcal{C}_{K}^{\prime}$ is empty whereas $S A T\left(\mathcal{C}_{T} \oplus \subseteq \mathcal{C}_{K}\right)$ is PSPACE-hard.

We write $\mathcal{L}_{1} \oplus \subset \mathcal{L}_{2}$ to mean the set $\mathcal{C}_{\mathcal{L}_{1}} \oplus \subset \mathcal{C}_{\mathcal{L}_{2}}$ for any monomodal logic $\mathcal{L}_{1}, \mathcal{L}_{2}$. By way of example, $S 5 \oplus \subseteq S 5$ equals $\mathcal{C}_{S 5} \oplus \subseteq \mathcal{C}_{S 5}$ but not $\mathcal{C}_{S 5}^{\prime} \oplus \subseteq \mathcal{C}_{S 5}^{\prime}$.

Lemma 1. For $\left\langle\mathcal{L}_{1}, \mathcal{L}_{2}\right\rangle \in(\{K, T, B, S 4, S 5\} \times\{K, T, B, S 4\}) \cup\langle S 4, S 5\rangle$, the problem $S A T\left(\mathcal{L}_{1} \oplus \subseteq \mathcal{L}_{2}\right)$ is PSPACE-hard.

We invite the reader to consult [9] for further topics on modal logic.

\section{PSPACE Ladner-like algorithms}

In this section, we show that $S A T\left(\mathcal{L}_{1} \oplus \subseteq \mathcal{L}_{2}\right)$ is in PSPACE for $\left\langle\mathcal{L}_{1}, \mathcal{L}_{2}\right\rangle \in$ $\{S 4, S 5\} \times\{T, B, S 4, S 5\}$. Observe that $\overline{S 4} \oplus \subseteq K=S 4 \oplus \subseteq T$ and $S 5 \oplus \subseteq K=$ $S 5 \oplus \subset T$. In the rest of this section we assume that $\mathcal{L}_{1} \in\{S 4, S 5\}$ and $\mathcal{L}_{2} \in$ $\{T, B, S 4, S 5\}$. We shall define Ladner-like algorithms.

\subsection{Preliminaries}

We introduce a (very simple) closure operator for sets of bimodal formulae. Let $X$ be a set of bimodal formulae. Let $s u b(X)$ be the smallest set of formulae including $X$, closed under subformulae and such that if $[2] \phi \in s u b(X)$, then $[1] \phi \in \operatorname{sub}(X)$. A set $X$ of formulae is said to be $s u b$-closed $\stackrel{\text { def }}{\Leftrightarrow} \operatorname{sub}(X)=X$. Observe that for any finite set $X$ of formulae, $m d(\operatorname{sub}(X))=m d(X)$ and for any formula $\phi, \operatorname{card}(\operatorname{sub}(\{\phi\}))<2 \times|\phi|$.

In order to determine the satisfiability of some formula $\phi$, we need to handle sets of formulae. Actually all those sets shall be subsets of $\operatorname{sub}(\{\phi\})$ and that is why $\operatorname{sub}(\{\phi\})$ has been introduced. In establishing the PSPACE complexity upper bound, the fact that not only $\operatorname{sub}(\{\phi\})$ is finite but also its cardinality is polynomial in the size of $\phi$ plays an important role. In the present case, the cardinality of $\operatorname{sub}(\{\phi\})$ is even linear in $|\phi|$.

In order to check whether $\phi$ is $\mathcal{L}_{1} \oplus \subset \mathcal{L}_{2}$-satisfiable, we build sequences of the form $X_{0} x_{0} X_{1} x_{1} X_{2} x_{2} \ldots$ where $\bar{\phi} \in X_{0} \subseteq \operatorname{sub}(\{\phi\})$ and for $i \in \omega, X_{i}$ is a consistent subset of $\operatorname{sub}(\{\phi\})$ and $x_{i} \in\{1,2\}$. We extend a finite sequence $X_{0} x_{0} X_{1} x_{1} \ldots x_{i-1} X_{i}$ with $x_{i} X_{i+1}$ whenever we need a witness of $\left[x_{i}\right] \psi \notin X_{i}$ for some formula $\psi$ (and $\psi \notin X_{i+1}$ ). The intention is to build paths in some 
$\mathcal{L}_{1} \oplus \subseteq \mathcal{L}_{2}$-model $\mathcal{M}=\left\langle W, R_{1}, R_{2}, m\right\rangle$ such that for $i \in \omega$, there is $w_{i} \in W$ such that $\mathcal{M}, w_{i} \models \psi$ iff $\psi \in X_{i}$ and $\left\langle w_{i}, w_{i+1}\right\rangle \in R_{x_{i}}$.

In order to establish termination which is a necessary step to obtain the PSPACE complexity upper bound, we shall define subsets $\operatorname{sub}(s, \phi) \subseteq \operatorname{sub}(\{\phi\})$ for $s \in\{1,2\}^{*}$ such that for $i \in \omega, X_{i} \subseteq \operatorname{sub}\left(x_{0} \ldots x_{i-1}, \phi\right)$. For $x_{i} \in\{1,2\}$, $\operatorname{sub}\left(x_{0} \ldots x_{i-1} \cdot x_{i}, \phi\right)$ contains all the formulae $\psi$ which we could possibly be put in $X_{i+1}$ for $\psi \in \operatorname{sub}\left(x_{0} \ldots x_{i-1}, \phi\right)$.

We are on the good track to get termination if there is some computable map $f: \omega \rightarrow \omega$ such that for $|s| \geq f(|\phi|), s u b(s, \phi)=\emptyset$. To establish the PSPACE complexity upper bound, $f$ should preferably be bounded by a polynomial. Those general principles may look quite attractive but in concrete examples of bimodal logics they are seldom sufficient to show that the satisfiability problem is in PSPACE. In $S 4 \oplus \subseteq S 4$, since transitivity of $R_{2}$ is required, if [2] $\psi \in X_{i}$, then $\mathcal{M}, w_{i} \vDash[2] \psi, \mathcal{M}, w_{i} \vDash[2][2] \psi$ and therefore one can expect that [2] $\psi \in X_{i+1}$ if $x_{i}=2$. So the formula [2] $\psi \in X_{i}$ should be propagated for any "2" transition. However, this does not guarantee termination. Actually, as already known from $[22,31,8]$, duplicates can be identified in $X_{0} x_{0} X_{1} x_{1} X_{2} x_{2} \ldots$ which corresponds to a cycle detection (see also [13]). Since $\operatorname{card}(\mathcal{P}(\operatorname{sub}(\{\phi\})))$ is in $\mathcal{O}\left(2^{|\phi|}\right)$, a finer analysis is necessary to establish the PSPACE complexity upper bound as done in [22] (see also [31] for the tense extension of Ladner's solution).

In order to conclude this introductory part that motivates the existence of the sets of the form $\operatorname{sub}(s, \phi)$, let us say that once the set $X_{i}$ of formulae is built and $x_{i}$ is chosen, the set $X_{i+1}$ of formulae satisfies the following conditions: $X_{i+1}$ is a consistent subset of $\operatorname{sub}\left(x_{0} \ldots x_{i}, \phi\right)$ and $\left\langle X_{i}, X_{i+1}\right\rangle$ satisfies a syntactic condition $\mathrm{C}_{x_{i}}$ that guarantees that $\mathcal{M}$ is an $\mathcal{L}_{1} \oplus \subset \mathcal{L}_{2}$-model and $\left\langle w_{i}, w_{i+1}\right\rangle \in R_{x_{i}}$.

Let $\phi$ be a bimodal formula. For $s \in\{1,2\}^{*}$, let $\operatorname{sub}(s, \phi)$ be the smallest set such that:

1. $\operatorname{sub}(\lambda, \phi)=\operatorname{sub}(\{\phi\}) ; \operatorname{sub}(s, \phi)$ is sub-closed;

2. if $[i] \psi \in \operatorname{sub}(s, \phi)$ for some $i \in\{1,2\}$, then $\psi \in \operatorname{sub}(s . i, \phi)$;

3. if $[1] \psi \in \operatorname{sub}(s, \phi)$, then $[1] \psi \in \operatorname{sub}(s .1, \phi)$;

4. if $\mathcal{L}_{2} \in\{S 4, S 5\}$ and [2] $\psi \in \operatorname{sub}(s, \phi)$, then $[2] \psi \in \operatorname{sub}(s .2, \phi)$ and $[2] \psi \in$ $\operatorname{sub}(s \cdot 1, \phi)$.

Observe that for any initial substring $s^{\prime}$ of the string $s \in\{1,2\}^{*}, \operatorname{sub}(s, \phi) \subseteq$ $\operatorname{sub}\left(s^{\prime}, \phi\right)$; for $k \geq 1, \operatorname{sub}(s \cdot 1, \phi)=\operatorname{sub}\left(s \cdot 1^{k}, \phi\right)$ and if $\mathcal{L}_{2} \in\{T, B\}$ and $s$ contains more than $k \geq m d(\phi)+1$ occurrences of 2 , then $\operatorname{sub}(s, \phi)=\emptyset$.

Definition 2. Let $X, Y$ be subsets of $\operatorname{sub}(\{\phi\})$. The binary relation $\mathrm{C}_{1}$ on the set $\mathcal{P}(\operatorname{sub}(\{\phi\}))$ is defined as follows: $X \mathrm{C}_{1} Y \stackrel{\text { gef }}{\Leftrightarrow}$

1.1. $\mathcal{L}_{2} \in\{T, B\}: X \mathrm{C}_{2} Y$ (see below);

1.2. $\mathcal{L}_{2} \in\{S 4, S 5\}$ : for all $[2] \psi \in X,[2] \psi \in Y$ and for all $[2] \psi \in Y,[2] \psi \in X$;

2.1. $\mathcal{L}_{1}=S 4$ : for all [1] $\psi \in X,[1] \psi \in Y$;

2.2. $\mathcal{L}_{1}=S 5$ : for all [1] $\psi \in X,[1] \psi \in Y$ and for all $[1] \psi \in Y,[1] \psi \in X$.

The binary relation $\mathrm{C}_{2}$ on $\mathcal{P}(\operatorname{sub}(\{\phi\}))$ is defined as follows: $X \mathrm{C}_{2} Y \stackrel{\text { def }}{\Leftrightarrow}$ 
1. $\mathcal{L}_{2}=T$ : for all $[2] \psi \in X, \psi \in Y$;

2. $\mathcal{L}_{2}=B$ : for all [2] $\psi \in X, \psi \in Y$ and for all [2] $\psi \in Y, \psi \in X$;

3. $\mathcal{L}_{2}=S 4$ : for all [2] $\psi \in X,[2] \psi \in Y$;

4. $\mathcal{L}_{2}=S 5$ : for all [2] $\psi \in X,[2] \psi \in Y$ and for all [2] $\psi \in Y,[2] \psi \in X$.

Let clos be the set of subsets $Y$ of $\operatorname{sub}(\{\phi\})$ such that for $i \in\{1,2\},[i] \psi \in$ $Y$ implies $\psi \in Y$. Observe that if $\mathcal{L}_{i} \in\{T, B, S 4, S 5\}$ [resp. $\mathcal{L}_{i} \in\{B, S 5\}$, $\mathcal{L}_{i} \in\{S 4, S 5\}$ ], then $\mathrm{C}_{i}$ restricted to clos is reflexive [resp. $\mathrm{C}_{i}$ is symmetric, $\mathrm{C}_{i}$ is transitive]. The logic $\mathcal{L}_{1}$ is anyhow in $\{S 4, S 5\}$ throughout this section. The careful reader may be puzzled by the point 1.2 . in the definition of $\mathrm{C}_{1}$ when $\mathcal{L}_{2}=S 4$. Indeed, this seems to give an S5 flavour to [2]. However, observe that for any bimodal frame $\left\langle W, R_{1}, R_{2}\right\rangle$ such that $R_{1}$ is symmetric, $R_{2}$ is transitive and $R_{1} \subseteq R_{2}$, for $\left\langle w, w^{\prime}\right\rangle \in R_{1}$, we have $R_{2}(w)=R_{2}\left(w^{\prime}\right)$ which implies that $w$ and $w^{\prime}$ satisfy the same set of [2]-formulae in any model based on $\left\langle W, R_{1}, R_{2}\right\rangle$. This gives us an additional reason to present the Ladner-like constructions for the logics studied in this section since this provides a rather uniform presentation.

Let $X$ be a subset of $s u b(s, \phi)$ for some $s \in\{1,2\}^{*}$ and for some formula $\phi$. The set $X$ is said to be $s$-consistent $\stackrel{\text { gef }}{\Leftrightarrow}$ for $\psi \in s u b(s, \phi)$ :

1. if $\psi=\neg \varphi$, then $\varphi \in X$ iff not $\psi \in X$;

2. if $\psi=\varphi_{1} \wedge \varphi_{2}$, then $\left\{\varphi_{1}, \varphi_{2}\right\} \subseteq X$ iff $\psi \in X$;

3. if $\psi=[i] \varphi$ for some $i \in\{1,2\}$ [resp. $\psi=[2] \varphi]$ and $\psi \in X$, then $\varphi \in X$ [resp. $[1] \varphi \in X]$.

Roughly speaking, the $s$-consistency entails the maximal propositional consistency with respect to $\operatorname{sub}(s, \phi)$. The condition 3 . above takes into account reflexivity and the inclusion $R_{1} \subseteq R_{2}$.

Lemma 3. Let $\mathcal{M}=\left\langle W, R_{1}, R_{2}, m\right\rangle$ be an $\mathcal{L}_{1} \oplus \subseteq \mathcal{L}_{2}$-model, $w, w^{\prime} \in W, s \in$ $\{1,2\}^{*}, i, i^{\prime} \in\{\lambda, 1,2\}$ and $\phi$ be a bimodal formula. Let $X_{w} \stackrel{\text { def }}{=}\{\psi \in \operatorname{sub}(s . i, \phi)$ : $\mathcal{M}, w \models \psi\}$ and $X_{w^{\prime}} \stackrel{\text { def }}{=}\left\{\psi \in \operatorname{sub}\left(s . i^{\prime}, \phi\right): \mathcal{M}, w^{\prime} \models \psi\right\}$. Then, $X_{w}$ is s.iconsistent, $X_{w^{\prime}}$ is s. $i^{\prime}$-consistent and if $\left\langle i, i^{\prime}\right\rangle \in\{\langle\lambda, \lambda\rangle,\langle\lambda, j\rangle\}$ and $\left\langle w, w^{\prime}\right\rangle \in R_{j}$ for some $j \in\{1,2\}$, then $X_{w} \mathrm{C}_{j} X_{w^{\prime}}$.

The proof of Lemma 3 is by an easy verification.

Lemma 4. Let $X_{i}$ be an $s_{i}$-consistent set and $s_{i} \in\{1,2\}^{*}, i=1,2$. Then,

(I) $\mathcal{L}_{1}=S 4: X_{1} \mathrm{C}_{1}^{*} X_{2}$ and $[2] \psi \in X_{1}$ implies $\psi \in X_{2}$;

(II) $\mathcal{L}_{1}=S 5: X_{1}\left(\mathrm{C}_{1} \cup \mathrm{C}_{1}^{-1}\right)^{*} X_{2}$ and $[2] \psi \in X_{1}$ implies $\psi \in X_{2}$;

(III) $\mathcal{L}_{2}=S 4: X_{1}\left(\mathrm{C}_{1} \cup \mathrm{C}_{2}\right)^{*} X_{2}$ and $[2] \psi \in X_{1}$ implies $\psi \in X_{2}$;

(IV) $\mathcal{L}_{2}=S 5: X_{1}\left(\mathrm{C}_{1} \cup \mathrm{C}_{1}^{-1} \cup \mathrm{C}_{2} \cup \mathrm{C}_{2}^{-1}\right)^{*} X_{2}$ and $[2] \psi \in X_{1}$ implies $\psi \in X_{2}$.

\subsection{The algorithms}

In Figure 1, the function wORLD $(\Sigma, s, \phi)$ returning a Boolean is defined. $\Sigma$ is a finite non-empty list of subsets of $\operatorname{sub}(\{\phi\})$ and $s \in\{1,2\}^{*}$. Moreover, for any 
function WORLD $(\Sigma, s, \phi)$

if last $(\Sigma)$ is not $s$-consistent, then return false;

for $[1] \psi \in \operatorname{sub}(s, \phi) \backslash \operatorname{last}(\Sigma)$ do

if there is no $X \in \Sigma$ such that $\Sigma=\Sigma_{1} X \Sigma_{2}, s$ is of the form $s_{1} . s_{2}$ with

$\left|s_{2}\right|=\left|\Sigma_{2}\right|$ and $s_{2} \in\{1\}^{*}, \psi \notin X$, last $(\Sigma) \mathrm{C}_{1} X$, then

for each $X_{\psi} \subseteq \operatorname{sub}(s .1, \phi) \backslash\{\psi\}$ such that $\operatorname{last}(\Sigma) \mathrm{C}_{1} X_{\psi}$, call WORLD $\left(\Sigma . X_{\psi}, s .1, \phi\right)$. If all these calls return false, then return false;

for $[2] \psi \in \operatorname{sub}(s, \phi) \backslash \operatorname{last}(\Sigma)$ do

$\mathcal{L}_{2} \in\{T, B\}$ : for each $X_{\psi} \subseteq \operatorname{sub}(s .2, \phi) \backslash\{\psi\}$ such that $\operatorname{last}(\Sigma) \mathrm{C}_{2} X_{\psi}$, call WORLD $\left(\Sigma . X_{\psi}, s .2, \phi\right)$. If all these calls return false, then return false;

$\mathcal{L}_{2} \in\{S 4, S 5\}$ : if there is no $X \in \Sigma$ such that $\psi \notin X$ and last $(\Sigma) \mathrm{C}_{2} X$, then

for each $X_{\psi} \subseteq \operatorname{sub}(s .2, \phi) \backslash\{\psi\}$ such that last $(\Sigma) \mathrm{C}_{2} X_{\psi}$, call WORLD $\left(\Sigma . X_{\psi}, s .2, \phi\right)$. If all these calls return false, then return false;

Return true.

Fig. 1. Algorithm WORLD

$X \subseteq \operatorname{sub}(\{\phi\})$ and for any call $\operatorname{WORLD}(\Sigma, s, \phi)$ in $\operatorname{WORLD}(X, \lambda, \phi)$ (at any recursion depth), last $(\Sigma) \subseteq \operatorname{sub}(s, \phi)$. The function WORLD is actually defined on the model of the function K-WORLD in [22] (see also [31,26, 35]).

Most of the ingenuity to guarantee that the algorithms terminate are in the definition of $s u b(s, \phi), s$-consistency and the conditions $\mathrm{C}_{i}$. Indeed, $s u b(s . i, \phi)$ contains the formulae that can be possibly propagated from $s u b(s, \phi)$. In the easiest case, $\operatorname{sub}(s . i, \phi) \subset \operatorname{sub}(s, \phi)$ but this is not the general case here. Then $\mathrm{C}_{i}$ and $s$-consistency further restrict the formulae that can be propagated. Still, we may be in trouble to guarantee termination. That is why the detection of cycles is introduced (see e.g. [22]). It is precisely, the appropriate combination of all these ingredients that guarantees termination and in the best case the PSPACE upper bound. What we present is a uniform formalization of Ladnerlike algorithms based on [31] and we believe it is the proper framework to allow further extensions.

We prove that for any set $X \subseteq \operatorname{sub}(\{\phi\}), \operatorname{WORLD}(X, \lambda, \phi)$ always terminates and requires polynomial space in $|\phi|$. We shall take advantage of the fact that if WORLD $(\Sigma, s, \phi)$ calls WORLD $\left(\Sigma^{\prime}, s^{\prime}, \phi\right)$ (at any recursion depth), then $\left|s^{\prime}\right|>|s|$.

Each subset $X \subseteq \operatorname{sub}(\{\phi\})$ can be represented as a bitstring of length $2 \times|\phi|$. By implementing $\bar{\Sigma}$ as a global stack, each level of the recursion uses space in $\mathcal{O}(|\phi|)$. For instance, in the parts of wORLD of the form "for each $X_{\psi} \subseteq \operatorname{sub}(s . i, \phi) \backslash$ $\{\psi\}$ such that $\operatorname{last}(\Sigma) \mathrm{C}_{i} X_{\psi}$, call WORLD $\left(\Sigma . X_{\psi}, s . i, \phi\right)$. If all these calls return false, then return false" the implementation uses a bitstring of length $2 \times|\phi|$ to encode $X_{\psi}$ (this value is incremented for each new $X_{\psi}$ ) and a Boolean indicating whether there is a call returning true.

Theorem 5. Let $\left\langle\mathcal{L}_{1}, \mathcal{L}_{2}\right\rangle \in\{S 4, S 5\} \times\{T, B, S 4, S 5\}$ and $n^{\prime}$ be the number of occurrences of $S 4$ in $\left\langle\mathcal{L}_{1}, \mathcal{L}_{2}\right\rangle$. Let $X \subseteq \operatorname{sub}(\{\phi\})$.

(I) WORLD $(X, \lambda, \phi)$ terminates and requires at most space in $\mathcal{O}\left(|\phi|^{3+n^{\prime}}\right)$; 
(II) Let WORLD $(\Sigma, s, \phi)$ be a call in the computation of WORLD $(X, \lambda, \phi)$. Then, $|\Sigma| \leq \alpha$ and $|s| \leq \alpha$ with $\alpha=(2 \times|\phi|+1)^{2-n^{\prime}} \times\left(4 \times|\phi|^{2}+1\right)^{n^{\prime}}$.

The bounds in Theorem 5 are really rough since many optimizations can be designed. Because of lack of place, this is omitted here.

Theorem 5 is certainly an important step to prove that satisfiability is in PSPACE but this is not sufficient. Indeed, until now we have no guarantee that WORLD is actually correct. This shall be shown in the next two lemmas.

Lemma 6. Let $\phi$ be a bimodal formula and $Y \subseteq s u b(\{\phi\})$ such that $\phi \in Y$. If WORLD $(Y, \lambda, \phi)$ returns true, then $\phi$ is $\mathcal{L}_{1} \oplus \subseteq \mathcal{L}_{2}$-satisfiable.

Proof. Let $n^{\prime}$ be the number of $S 4$ in $\left\langle\mathcal{L}_{1}, \mathcal{L}_{2}\right\rangle$. Assume that WORLD $(Y, \lambda, \phi)$ returns true. Let us build an $\mathcal{L}_{1} \oplus \subset \mathcal{L}_{2}$-model $\mathcal{M}=\left\langle W, R_{1}, R_{2}, m\right\rangle$ for which there is $w \in W$ such that for all $\psi \in \operatorname{sub}(\{\phi\}), \mathcal{M}, w \models \psi$ iff $\psi \in Y$.

Let $S$ be the set of strings $s$ in $\{1,2\}^{*}$ such that $|s| \leq(2 \times|\phi|+1)^{2-n^{\prime}} \times(4 \times$ $\left.|\phi|^{2}+1\right)^{n^{\prime}}$. We define $W$ as the set of pairs $\langle X, s\rangle \in \operatorname{clos} \times S$ for which there is a finite sequence $\left\langle\Sigma_{1}, s_{1}\right\rangle, \ldots,\left\langle\Sigma_{k}, s_{k}\right\rangle(k \geq 1)$ such that

1. $\Sigma_{1}=Y ; s_{1}=\lambda ; \operatorname{last}\left(\Sigma_{k}\right)=X ; s_{k}=s$;

2. for $i \in\{1, \ldots, k\}$, WORLD $\left(\Sigma_{i}, s_{i}, \phi\right)$ returns true;

3. for $i \in\{1, \ldots, k-1\}$, WORLD $\left(\Sigma_{i}, s_{i}, \phi\right)$ calls directly WORLD $\left(\Sigma_{i+1}, s_{i+1}, \phi\right)$.

The conditions 2 . and 3 . state that we only record the pairs $\langle X, s\rangle \in \operatorname{clos} \times S$ that contribute to make WORLD $(Y, \lambda, \phi)$ true. $\langle Y, \lambda\rangle \in W$ by definition. Furthermore, for all $\langle X, s\rangle \in W, X \subseteq \operatorname{sub}(s, \phi)$ and $X$ is $s$-consistent.

Let us define the auxiliary binary relations $R_{1}^{\prime}$ [resp. $R_{2}^{\prime}$ ] on $W$ as follows: $\langle X, s\rangle R_{1}^{\prime}\left\langle X^{\prime}, s^{\prime}\right\rangle$ [resp. $\left.\langle X, s\rangle R_{2}^{\prime}\left\langle X^{\prime}, s^{\prime}\right\rangle\right] \stackrel{\text { def }}{\Leftrightarrow}$ there is a call wORLD $(\Sigma, s, \phi)$ in WORLD $(Y, \lambda, \phi)$ (at any depth of the recursion) such that

1. either

(a) $\operatorname{last}(\Sigma)=X$;

(b) WORLD $(\Sigma, s, \phi)$ calls WORLD $\left(\Sigma^{\prime}, s^{\prime}, \phi\right)$ in the "1" [resp. "2"] segment of WORLD $(\Sigma, s, \phi) ;$ last $\left(\Sigma^{\prime}\right)=X^{\prime}$

2. or there is a finite sequence $\left\langle\Sigma_{1}, s_{1}\right\rangle, \ldots,\left\langle\Sigma_{k}, s_{k}\right\rangle$ such that:

(a) $\operatorname{last}\left(\Sigma_{k}\right)=X ; \operatorname{last}\left(\Sigma_{1}\right)=X^{\prime} ; \Sigma_{k}=\Sigma ; s_{k}=s ; s_{1}=s^{\prime}$;

(b) for $i \in\{1, \ldots, k\}$, $\left\langle\right.$ last $\left.\left(\Sigma_{i}\right), s_{i}\right\rangle \in W$;

(c) for $i \in\{1, \ldots, k-1\}$, WORLD $\left(\Sigma_{i}, s_{i}, \phi\right)$ calls WORLD $\left(\Sigma_{i+1}, s_{i+1}, \phi\right)$ in the "1" [resp. in either the "1" or the "2"] segment of wORLD;

(d) the call WORLD $\left(\Sigma_{k}, s_{k}, \phi\right)$ enters in the "1" [resp. "2"] segment of wORLD and for some formula $[1] \psi \in \operatorname{sub}(s, \phi) \backslash X[\operatorname{resp} .[2] \psi \in \operatorname{sub}(s, \phi) \backslash X]$, no recursive call to WORLD is necessary thanks to $\Sigma_{1}, \psi \notin X^{\prime}, X \mathrm{C}_{1} X^{\prime}$ [resp. $\left.X \mathrm{C}_{2} X^{\prime}\right]$.

If $\mathcal{L}_{2} \in\{T, B\}$, the second possibility in the definition of $R_{2}^{\prime}$ above should not be taken into account. The definition of $\mathcal{M}$ can be now completed:

$-\mathcal{L}_{1}=S 4: R_{1} \stackrel{\text { def }}{=}\left(R_{1}^{\prime}\right)^{*} ; \mathcal{L}_{1}=S 5: R_{1} \stackrel{\text { def }}{=}\left(R_{1}^{\prime} \cup R_{1}^{\prime-1}\right)^{*} ;$ 
$-\mathcal{L}_{2}=T: R_{2} \stackrel{\text { def }}{=} R_{1} \cup R_{2}^{\prime} ; \mathcal{L}_{2}=B: R_{2} \stackrel{\text { def }}{=} R_{1} \cup R_{2}^{\prime} \cup\left(R_{1}\right)^{-1} \cup\left(R_{2}^{\prime}\right)^{-1}$;

$-\mathcal{L}_{2}=S 4: R_{2} \stackrel{\text { def }}{=}\left(R_{1} \cup R_{2}^{\prime}\right)^{*} ; \mathcal{L}_{2}=S 5: R_{2} \stackrel{\text { def }}{=}\left(R_{1} \cup R_{2}^{\prime} \cup\left(R_{1}\right)^{-1} \cup\left(R_{2}^{\prime}\right)^{-1}\right)^{*}$;

- for $\mathrm{p} \in$ For $_{0}, m(\mathrm{p}) \stackrel{\text { def }}{=}\{\langle X, s\rangle \in W: \mathrm{p} \in X\}$.

$\mathcal{M}$ is an $\mathcal{L}_{1} \oplus \subseteq \mathcal{L}_{2}$-model. One can show (i) $\langle X, s\rangle R_{1}^{\prime}\left\langle X^{\prime}, s^{\prime}\right\rangle$ implies $X \mathrm{C}_{1} X^{\prime}$ and (ii) $\langle X, s\rangle R_{2}^{\prime}\left\langle X^{\prime}, s^{\prime}\right\rangle$ implies $X \mathrm{C}_{2} X^{\prime}$. So, (iii) for $j \in\{1,2\},\langle X, s\rangle R_{j}\left\langle X^{\prime}, s^{\prime}\right\rangle$ implies for all $[j] \psi \in X, \psi \in X^{\prime}$ (by Lemma 4). By induction on the structure of $\psi$ we show that for all $\langle X, s\rangle \in W$, for all $\psi \in \operatorname{sub}(s, \phi), \psi \in X$ iff $\mathcal{M},\langle X, s\rangle \models \psi$. The case when $\psi$ is a propositional variable is by definition of $m$.

Induction Hypothesis: for all $\psi \in \operatorname{sub}(\phi)$ such that $|\psi| \leq n$, for all $\langle X, s\rangle \in W$, if $\psi \in \operatorname{sub}(s, \phi)$, then $\psi \in X$ iff $\mathcal{M},\langle X, s\rangle \models \psi$.

Let $\psi$ be a formula in $\operatorname{sub}(\phi)$ such that $|\psi| \leq n+1$. The cases when the outermost connective of $\psi$ is Boolean is a consequence of the s-consistency of $X$ and the induction hypothesis. Let us treat the other cases.

Case 1: $\psi=[1] \psi^{\prime}$. Let $\langle X, s\rangle \in W$ such that $\psi \in \operatorname{sub}(s, \phi)$. By definition of $W$, there is $\Sigma$ such that last $(\Sigma)=X$ and $\operatorname{WORLD}(\Sigma, s, \phi)$ returns true. If $\psi \in X$, then by (iii), for all $\left\langle X^{\prime}, s^{\prime}\right\rangle \in R_{1}(\langle X, s\rangle), \psi^{\prime} \in X^{\prime}$. One can show that $\psi^{\prime} \in \operatorname{sub}\left(s^{\prime}, \phi\right)$. By the induction hypothesis, $\mathcal{M},\left\langle X^{\prime}, s^{\prime}\right\rangle \vDash \psi^{\prime}$ and therefore $\mathcal{M},\langle X, s\rangle \vDash \psi$. Now, if $\psi \notin X$, two cases are distinguished.

Case 1.1: there is $X^{\prime}$ in $\Sigma$ such that $X \mathrm{C}_{1} X^{\prime}, \psi^{\prime} \notin X^{\prime}$ and $\Sigma=\Sigma^{\prime} X^{\prime} \Sigma_{2}, s$ is of the form $s^{\prime} . s_{2}$ with $\left|\Sigma_{2}\right|=\left|s_{2}\right|$ and $s_{2} \in\{1\}^{*}$. By definition of $W$, WORLD $\left(\Sigma^{\prime} . X^{\prime}, s^{\prime}, \phi\right)$ returns true (see the conditions 2. and 3. defining $W$ ). Hence, $\langle X, s\rangle R_{1}^{\prime}\left\langle X^{\prime}, s^{\prime}\right\rangle$ by definition and therefore $\langle X, s\rangle R_{1}\left\langle X^{\prime}, s^{\prime}\right\rangle$. One can show that $\psi^{\prime} \in s u b\left(s^{\prime}, \phi\right)$ since $s$ is of the form $s^{\prime} \cdot 1^{k}$ for some $k \geq 0$. By induction hypothesis, $\mathcal{M},\left\langle X^{\prime}, s^{\prime}\right\rangle \not \psi^{\prime}$ and therefore $\mathcal{M},\langle X, s\rangle \forall \psi$.

Case 1.2: WORLD $(\Sigma, s, \phi)$ calls successfully WORLD $\left(\Sigma^{\prime}, s^{\prime}, \phi\right)$ in the "1" segment of WORLD, last $\left(\Sigma^{\prime}\right)=X^{\prime}$ and $\psi^{\prime} \notin \operatorname{last}\left(\Sigma^{\prime}\right), X \mathrm{C}_{1} X^{\prime}$, and $X^{\prime} \subseteq \operatorname{sub}\left(s^{\prime}, \phi\right)$. Moreover, we have $s^{\prime}=s .1$. This is so since WORLD $(\Sigma, s, \phi)$ returns true. By definition of $R_{1}^{\prime},\langle X, s\rangle R_{1}^{\prime}\left\langle X^{\prime}, s^{\prime}\right\rangle$. Furthermore, one can easily show that $\psi^{\prime} \in \operatorname{sub}\left(s^{\prime}, \phi\right)$. By the induction hypothesis, $\mathcal{M},\left\langle X^{\prime}, s^{\prime}\right\rangle \forall \psi^{\prime}$ and therefore $\mathcal{M},\langle X, s\rangle \forall \psi$.

Case 2: $\psi=[2] \psi^{\prime}$. This is analogous to the Case 1.

As a conclusion, since $\phi \in Y$ and WORLD $(Y, \lambda, \phi)$ returns true, $\mathcal{M},\langle Y, \lambda\rangle \vDash \phi$ and therefore $\phi$ is $\mathcal{L}_{1} \oplus \subseteq \mathcal{L}_{2}$-satisfiable.

The proof of Lemma 6 can be viewed as a way to transform a successful call $\operatorname{WORLD}(Y, \lambda, \phi)$ into a quasi $\mathcal{L}_{1} \oplus \subset \mathcal{L}_{2}$-model by analyzing the computation tree of WORLD $(Y, \lambda, \phi)$. Then, this quasi $\mathcal{L}_{1} \oplus \subset \mathcal{L}_{2}$-model is appropriately completed in order to get an $\mathcal{L}_{1} \oplus \subset \mathcal{L}_{2}$-model. The idea to construct a (standard) model from different coherent pieces is very common to establish decidability and complexity results for modal logics (see e.g. $[22,28,5,23])$. Mosaics technique uses such an approach (see e.g. [23]).

Lemma 7. Let $\phi$ be a bimodal formula. If $\phi$ is $\mathcal{L}_{1} \oplus \subseteq \mathcal{L}_{2}$-satisfiable, then there is $Y \subseteq \operatorname{sub}(\{\phi\})$ such that $\phi \in Y$ and $\operatorname{WORLD}(Y, \lambda, \phi)$ returns true.

Since WORLD is correct, the proof of Lemma 6 provides the finite model property for $\mathcal{L}_{1} \oplus \subseteq \mathcal{L}_{2}$ and an exponential bound for the size of the models exists. 
Theorem 8. For $\left\langle\mathcal{L}_{1}, \mathcal{L}_{2}\right\rangle \in\{S 4, S 5\} \times\{T, B, S 4, S 5\}, S A T\left(\mathcal{L}_{1} \oplus \subseteq \mathcal{L}_{2}\right)$ is in PSPACE.

For $\left\langle\mathcal{L}_{1}, \mathcal{L}_{2}\right\rangle \in\{S 4, S 5\} \times\{T, B, S 4\} \cup\langle S 4, S 5\rangle, S A T\left(\mathcal{L}_{1} \oplus \subset \mathcal{L}_{2}\right)$ is PSPACEhard. So, in particular $S A T(S 5 \oplus \subset S 4)$ is PSPACE-complete and the logic $\mathrm{S} 4+5$ introduced in [33] has consequently a PSPACE-complete satisfiability problem. Until now, we have not yet established that $S A T(S 5 \oplus \subset-S 5)$ is PSPACE-hard as $S A T(S 5 \oplus S 5)$ [18]. It is unlikely since by [11, Proposition 4.8] $S A T(S 5 \oplus \subseteq S 5)$ is NP-complete.

\section{Reduction into a PSPACE guarded fragment}

By FO2 we mean the fragment of first-order logic without equality or function symbols using only two variables. In this section we show that for $\mathcal{L}_{1}, \mathcal{L}_{2} \in$ $\{K, T, B\}, S A T\left(\mathcal{L}_{1} \oplus \subseteq \mathcal{L}_{2}\right)$ can be linearly translated into a known PSPACE fragment of FO2, say WLGF2 standing for weak loosely guarded fragment with two variables. The vocabulary of WLGF 2 consists of: the symbols $\neg, \wedge, \Rightarrow, \forall$ for propositional connectives and universal quantification; a countable set $\left\{\mathrm{P}_{i}: i \in\right.$ $\omega\}$ of unary predicate symbols; a set $\left\{R_{1}, R_{2}\right\}$ of binary predicate symbols and a set $\left\{\mathrm{x}_{0}, \mathrm{x}_{1}\right\}$ of individual variables. The set of WLGF2-formulae is the smallest set containing the set of atomic formulae built over this vocabulary, closed under the standard rules for Boolean connectives and under the rule below: if $\phi\left(\mathrm{x}_{i}\right)$ and $\psi\left(\mathbf{x}_{i}, \mathbf{x}_{1-i}\right)$ are WLGF2-formulae for some $i \in\{0,1\}$ such that,

- the only variable free in $\phi\left(\mathbf{x}_{i}\right)$ is $\mathbf{x}_{i}$;

$-\psi\left(\mathrm{x}_{i}, \mathrm{x}_{1-i}\right)$ is a conjunction of atomic formulae of the form $\mathrm{R}(\mathrm{x}, \mathrm{y})$ such that for at least one conjunct $\{\mathrm{x}, \mathrm{y}\}=\left\{\mathrm{x}_{0}, \mathrm{x}_{1}\right\}$;

then $\forall \mathrm{x}_{i}\left(\psi\left(\mathrm{x}_{i}, \mathrm{x}_{1-i}\right) \Rightarrow \phi\left(\mathrm{x}_{i}\right)\right)$ is a WLGF2-formula. WLGF2 is a fragment of the loosely guarded fragment LGF (see e.g. [4]). Actually WLGF2 is even a fragment of $\mathcal{P G} \mathcal{F}_{2}$ defined in [23] and shown to be in PSPACE [23]. None of the obvious FO2-formulae capturing reflexivity, symmetry and inclusion are WLGF2-formulae. Instead of using such axioms, we introduce $\mathcal{P G} \mathcal{F}_{2}$-modalities in the sense of [23, Section 4.1.1].

For $\mathcal{L}_{1}, \mathcal{L}_{2} \in\{K, T, B\}$, we define a map $T_{\mathcal{L}_{1} \oplus \subset \mathcal{L}_{2}}$ such that $T_{\mathcal{L}_{1} \oplus \subset \mathcal{L}_{2}}(\phi)$ is of the form init ${\text { L } 1 \oplus \subset \mathcal{L}_{2}} \wedge S T_{\mathcal{L}_{1} \oplus \subset \mathcal{L}_{2}}\left(\phi, \mathrm{x}_{0}\right)$ where init $_{\mathcal{L}_{1} \oplus \subset \mathcal{L}_{2}}$ is a fixed WLGF2formula. Analogously to the standard translation $S T$ [3], $S T_{\mathcal{L}_{1} \oplus \subset} \mathcal{L}_{2}$ encodes the quantification in the interpretation of $[i]$ into the language of WLGF2. We allow ourselves only a restricted form of universal quantification that encodes appropriately the properties of the bimodal frames. The main idea of $S T_{\mathcal{L}_{1} \oplus \subseteq \mathcal{L}_{2}}$ is to visit only the successor worlds that satisfy the local constraints on the relations of the frames. Indeed, reflexivity, symmetry and inclusion can be checked locally. $S T_{\mathcal{L}_{1} \oplus \subseteq \mathcal{L}_{2}}$ is defined inductively as follows $(i \in\{0,1\})$;

$-S T_{\mathcal{L}_{1} \oplus \subseteq \mathcal{L}_{2}}\left(\mathrm{p}_{j}, \mathrm{x}_{i}\right) \stackrel{\text { def }}{=} \mathrm{P}_{j}\left(\mathrm{x}_{i}\right) ;$

- $S T_{\mathcal{L}_{1} \oplus \subseteq \mathcal{L}_{2}}\left(\phi_{1} \wedge \phi_{2}, \mathbf{x}_{i}\right) \stackrel{\text { def }}{=} S T_{\mathcal{L}_{1} \oplus \subseteq \mathcal{L}_{2}}\left(\phi_{1}, \mathbf{x}_{i}\right) \wedge S T_{\mathcal{L}_{1} \oplus \subseteq \mathcal{L}_{2}}\left(\phi_{2}, \mathbf{x}_{i}\right) ;$ 
$-S T_{\mathcal{L}_{1} \oplus \subseteq \mathcal{L}_{2}}\left([j] \phi, \mathrm{x}_{i}\right) \stackrel{\text { def }}{=} \forall \mathrm{x}_{1-i}\left(\left(\bigwedge_{k \in\{j, 2\}} \mathrm{R}_{k}\left(\mathrm{x}_{i}, \mathrm{x}_{1-i}\right) \wedge \varphi_{\mathcal{L}_{1} \oplus \subseteq \mathcal{L}_{2}}^{k}\left(\mathrm{x}_{i}, \mathrm{x}_{1-i}\right)\right) \Rightarrow\right.$ $\left.S T_{\mathcal{L}_{1} \oplus \subseteq \mathcal{L}_{2}}\left(\phi, \mathrm{x}_{1-i}\right)\right)$ for $j \in\{1,2\}$ where the $\varphi_{\mathcal{L}_{1} \oplus \subseteq \mathcal{L}_{2}}^{k}\left(\mathrm{x}_{i}, \mathrm{x}_{1-i}\right)$ s are defined in the table below.

\begin{tabular}{|c|c|c|c|}
\hline \hline $\mathcal{L}_{1}$ & $\mathcal{L}_{2}$ & $\varphi_{\mathcal{L}_{1} \oplus \mathcal{C} \mathcal{L}_{2}}^{1}\left(\mathrm{x}_{i}, \mathrm{x}_{1-i}\right)$ & $\varphi_{\mathcal{L}_{1} \oplus \mathcal{C} \mathcal{L}_{2}}^{2}\left(\mathrm{x}_{i}, \mathrm{x}_{1-i}\right)$ \\
\hline $\mathrm{K}$ & $\mathrm{K}$ & $\mathrm{T}$ & $\mathrm{T}$ \\
\hline $\mathrm{K}$ & $\mathrm{T}$ & $\mathrm{T}$ & $\mathrm{R}_{2}\left(\mathrm{x}_{1-i}, \mathrm{x}_{1-i}\right)$ \\
\hline $\mathrm{K}$ & $\mathrm{B}$ & $\mathrm{T}$ & $\mathrm{R}_{2}\left(\mathrm{x}_{1-i}, \mathrm{x}_{i}\right) \wedge \mathrm{R}_{2}\left(\mathrm{x}_{1-i}, \mathrm{x}_{1-i}\right)$ \\
\hline $\mathrm{T}$ & $\mathrm{K}$ & $\mathrm{R}_{1}\left(\mathrm{x}_{1-i}, \mathrm{x}_{1-i}\right)$ & $\mathrm{R}_{1}\left(\mathrm{x}_{1-i}, \mathrm{x}_{1-i}\right)$ \\
\hline $\mathrm{T}$ & $\mathrm{T}$ & $\mathrm{R}_{1}\left(\mathrm{x}_{1-i}, \mathrm{x}_{1-i}\right)$ & $\mathrm{R}_{2}\left(\mathrm{x}_{1-i}, \mathrm{x}_{1-i}\right) \wedge \mathrm{R}_{1}\left(\mathrm{x}_{1-i}, \mathrm{x}_{1-i}\right)$ \\
\hline $\mathrm{T}$ & $\mathrm{B}$ & $\mathrm{R}_{1}\left(\mathrm{x}_{1-i}, \mathrm{x}_{1-i}\right)$ & $\mathrm{R}_{2}\left(\mathrm{x}_{1-i}, \mathrm{x}_{1-i}\right) \wedge \mathrm{R}_{1}\left(\mathrm{x}_{1-i}, \mathrm{x}_{1-i}\right) \wedge \mathrm{R}_{2}\left(\mathrm{x}_{1-i}, \mathrm{x}_{i}\right)$ \\
\hline $\mathrm{B}$ & $\mathrm{K}$ & $\mathrm{R}_{1}\left(\mathrm{x}_{1-i}, \mathrm{x}_{i}\right) \wedge \mathrm{R}_{1}\left(\mathrm{x}_{1-i}, \mathrm{x}_{1-i}\right)$ & $\mathrm{R}_{1}\left(\mathrm{x}_{1-i}, \mathrm{x}_{1-i}\right)$ \\
\hline $\mathrm{B}$ & $\mathrm{T}$ & $\mathrm{R}_{1}\left(\mathrm{x}_{1-i}, \mathrm{x}_{i}\right) \wedge \mathrm{R}_{1}\left(\mathrm{x}_{1-i}, \mathrm{x}_{1-i}\right)$ & $\mathrm{R}_{1}\left(\mathrm{x}_{1-i}, \mathrm{x}_{1-i}\right) \wedge \mathrm{R}_{2}\left(\mathrm{x}_{1-i}, \mathrm{x}_{1-i}\right)$ \\
\hline $\mathrm{B}$ & $\mathrm{B}$ & $\mathrm{R}_{1}\left(\mathrm{x}_{1-i}, \mathrm{x}_{i}\right) \wedge \mathrm{R}_{1}\left(\mathrm{x}_{1-i}, \mathrm{x}_{1-i}\right)$ & $\mathrm{R}_{2}\left(\mathrm{x}_{1-i}, \mathrm{x}_{1-i}\right) \wedge \mathrm{R}_{1}\left(\mathrm{x}_{1-i}, \mathrm{x}_{1-i}\right) \wedge \mathrm{R}_{2}\left(\mathrm{x}_{1-i}, \mathrm{x}_{i}\right)$ \\
\hline \hline
\end{tabular}

Let us define the initial formulae: init $_{K \oplus \subseteq K} \stackrel{\text { def }}{=} \top ;$ init $_{T \oplus \subseteq K} \stackrel{\text { def }}{=}$ init $_{B \oplus \subseteq K} \stackrel{\text { def }}{=}$ $\mathrm{R}_{1}\left(\mathrm{x}_{0}, \mathrm{x}_{0}\right) ;$ init $_{K \oplus \subseteq T} \stackrel{\text { def }}{=}$ init $_{K \oplus \subseteq B} \stackrel{\text { def }}{=} \mathrm{R}_{2}\left(\mathrm{x}_{0}, \mathrm{x}_{0}\right)$; for $\mathcal{L}_{1}, \mathcal{L}_{2} \in\{T, B\}$, init ${\dot{\mathcal{L}_{1} \oplus \subseteq}}_{\mathcal{L}_{2}}$ $\stackrel{\text { def }}{=} \mathrm{R}_{1}\left(\mathrm{x}_{0}, \mathrm{x}_{0}\right) \wedge \mathrm{R}_{2}\left(\mathrm{x}_{0}, \mathrm{x}_{0}\right)$.

Lemma 9. For $\mathcal{L}_{1}, \mathcal{L}_{2} \in\{K, T, B\}$, for any formula $\phi, \phi \in S A T\left(\mathcal{L}_{1} \oplus \subset \mathcal{L}_{2}\right)$ iff init $_{\mathcal{L}_{1} \oplus \subset \mathcal{L}_{2}} \wedge S T_{\mathcal{L}_{1} \oplus \subset \mathcal{L}_{2}}\left(\phi, \mathrm{x}_{0}\right)$ is WLGF多satisfiable.

Since $T_{\mathcal{L}_{1} \oplus \subseteq \mathcal{L}_{2}}$ is in linear-time,

Theorem 10. For $\mathcal{L}_{1}, \mathcal{L}_{2}$ in $\{K, T, B\}, S A T\left(\mathcal{L}_{1} \oplus \subseteq \mathcal{L}_{2}\right)$ is in PSPACE.

\section{EXPTIME-complete bimodal logics}

It remains to characterize the complexity of $S A T\left(\mathcal{L}_{1} \oplus \subseteq \mathcal{L}_{2}\right)$ for $\left\langle\mathcal{L}_{1}, \mathcal{L}_{2}\right\rangle \in$ $\{K, T, B\} \times\{S 4, S 5\}$. By using logarithmic space transformations into conversePDL (that is known to be in EXPTIME), for $\left\langle\mathcal{L}_{1}, \mathcal{L}_{2}\right\rangle \in\{K, T, B\} \times\{S 4, S 5\}$, $S A T\left(\mathcal{L}_{1} \oplus \subseteq \mathcal{L}_{2}\right)$ can be shown to be in EXPTIME. Let $\mathcal{C}$ be a class of monomodal frames. We write $G S A T(\mathcal{C})$ to denote the set of monomodal formulae $\phi$ such that there is a $\mathcal{C}$-model $\mathcal{M}=\left\langle W, R_{1}, m\right\rangle$ satisfying for all $w \in W, \mathcal{M}, w \models \phi$.

Lemma 11. Let $\mathcal{C}, \mathcal{C}^{\prime}$ be classes of monomodal frames such that $\mathcal{C}_{S 5} \subseteq \mathcal{C} \subseteq$ $\mathcal{C}_{K 4}$ and $\mathcal{C}^{\prime}$ is closed under generated subframes, disjoint unions and isomorphic copies. Then, for any monomodal formula $\phi, \phi \in G S A T\left(\mathcal{C}^{\prime}\right)$ iff $[2] \phi \wedge \phi \in$ $S A T\left(\mathcal{C}^{\prime} \oplus \subseteq \mathcal{C}\right)$.

Proof. The idea of the proof has its origin in the proof of [32, Proposition 7] where it is shown that the respective global satisfiability problems for $\mathrm{S} 4$ and S5 are identical, that is $G S A T(S 4)=G S A T(S 5)$. One can show that since $\mathcal{C}^{\prime}$ is closed under generated subframes, disjoint union and isomorphic copies, $S A T\left(\mathcal{C}^{\prime} \oplus \subseteq \mathcal{C}_{S 5}\right)=S A T\left(\mathcal{C}^{\prime} \oplus \subseteq \mathcal{C}_{S 5}^{\prime}\right)$. So in $\mathcal{C}^{\prime} \oplus \subseteq \mathcal{C}_{S 5}$, the modal connective [2] behaves as a universal modal connective. 
Let $\phi$ be a monomodal formula. Assume that $\phi \in G S A T\left(\mathcal{C}^{\prime}\right)$. So, there is an $\mathcal{C}^{\prime} \oplus \subset \mathcal{C}_{S 5}$-model $\mathcal{M}=\left\langle W, R_{1}, R_{2}, m\right\rangle$ and $w \in W$ such that $\mathcal{M}, w \models[2] \phi$. Since $R_{2}$ is reflexive, $\mathcal{M}, w \models[2] \phi \wedge \phi$. By hypothesis, $\mathcal{C}^{\prime} \oplus \subseteq \mathcal{C}_{S 5} \subseteq \mathcal{C}^{\prime} \oplus \subseteq \mathcal{C}$, so $[2] \phi \wedge \phi \in S A T\left(\mathcal{C}^{\prime} \oplus \subseteq \mathcal{C}\right)$.

Now assume that $[2] \phi \wedge \phi \in S A T\left(\mathcal{C}^{\prime} \oplus \subset \mathcal{C}\right)$. So, there is a $\mathcal{C}^{\prime} \oplus \subset \mathcal{C}$-model $\mathcal{M}=\left\langle W, R_{1}, R_{2}, m\right\rangle$ such that for some $w \in W, \mathcal{M}, w \models[2] \phi \wedge \phi$. Since $R_{2}$ is transitive, for $w^{\prime} \in R_{2}^{*}(w), \mathcal{M}, w^{\prime} \vDash \phi$. In particular, for $w^{\prime} \in R_{1}^{*}(w), \mathcal{M}, w^{\prime} \vDash \phi$. Let $\mathcal{M}=\left\langle W^{\prime}, R_{1}^{\prime}, R_{2}^{\prime}, m^{\prime}\right\rangle$ be the $\mathcal{C}^{\prime} \oplus \subseteq \mathcal{C}_{S 5}$-model such that $W^{\prime}=R_{1}^{*}(w)$ and, $R_{1}^{\prime}$ and $m^{\prime}$ are the respective restrictions of $R_{1}$ and $m$ to $W^{\prime}$ and $R_{2}^{\prime}=W^{\prime} \times W^{\prime}$. Since $\mathcal{C}^{\prime}$ is closed under generated subframes, $\left\langle W^{\prime}, R_{1}^{\prime}\right\rangle \in \mathcal{C}^{\prime}$. So, $\phi \in G S A T\left(\mathcal{C}^{\prime}\right)$.

Many examples of classes of frames between $\mathcal{C}_{K 4}$ and $\mathcal{C}_{S 5}$ can be found in [17, Figure 4].

Theorem 12. Let $\mathcal{L}_{1}, \mathcal{L}_{2}$ be monomodal logics such that $K \subseteq \mathcal{L}_{1} \subseteq B, K 4 \subseteq$ $\mathcal{L}_{2} \subseteq S 5$ and for $i \in\{1,2\}, \mathcal{L}_{i}$ is complete with respect to $\mathcal{C}_{\mathcal{L}_{i}}$. Then, $\overline{S A T}\left(\mathcal{C}_{\mathcal{L}_{1}} \oplus \underline{\subseteq}\right.$ $\left.\mathcal{C}_{\mathcal{L}_{2}}\right)$ is EXPTIME-hard.

Proof. By [10, Theorem 1] (see also [30]), GSAT( $\left.\mathcal{C}_{\mathcal{L}_{1}}\right)$ is EXPTIME-hard. By Lemma 11, $S A T\left(\mathcal{C}_{\mathcal{L}_{1}} \oplus \subseteq \mathcal{C}_{\mathcal{L}_{2}}\right)$ is EXPTIME-hard $\left(\mathcal{C}_{\mathcal{L}_{1}}\right.$ is closed under generated subframes, disjoint unions and isomorphic copies).

Hence, for $\left\langle\mathcal{L}_{1}, \mathcal{L}_{2}\right\rangle \in\{K, T, B\} \times\{S 4, S 5\}, S A T\left(\mathcal{L}_{1} \oplus \subset \mathcal{L}_{2}\right)$ is EXPTIMEhard. Since $K \oplus \subseteq S 4$ is a fragment of the logic $\mathcal{A}$ introduced in [7], $\mathcal{A}$-satisfiability is EXPTIME-hard. $\mathcal{A}$-satisfiability can be also translated in logarithmic space into PDL (see also [6]).

\section{Concluding remarks}

We have characterized the computational complexity of simple dependent bimodal logics. Table 1 summarizes the main results. As a side-effect, we have established that $\mathrm{S} 4+5$ [33] is PSPACE-complete whereas the logic $\mathcal{A}$ in [7] is EXPTIME-complete. Unlike the fusion operator $\oplus$, the situation with $\oplus \subset$ is not uniform since NP-complete, PSPACE-complete and EXPTIME-complete dependent bimodal logics have been found. The only case of NP-complete logic is $S 5 \oplus \subset S 5$ and we conjecture that this can be generalized to extensions of S4.3.

The most interesting proofs are related to PSPACE upper bounds. We used two proof techniques. The first one consists in translation $S A T\left(\mathcal{L}_{1} \oplus \subset \mathcal{L}_{2}\right)$ for $\mathcal{L}_{1}, \mathcal{L}_{2} \in\{K, T, B\}$ into satisfiability for a fragment of M. Marx's packed guarded fragment with only two individual variables $\mathcal{P} \mathcal{G} \mathcal{F}_{2}$. This approach has obvious limitations as soon as transitive relations are involved.

The second technique consists in defining Ladner-like decision procedures for $\left\langle\mathcal{L}_{1}, \mathcal{L}_{2}\right\rangle \in\{S 4, S 5\} \times\{T, B, S 4, S 5\}$ extending Ladner technique following [31] and we have presented a uniform framework that can be easily reused to study other polymodal logics. Indeed, it is the appropriate definitions of the sets $\operatorname{sub}(s, \phi)$, the notion of $s$-consistency, the conditions $\mathrm{C}_{i}$ and possibly the 
mechanism of cycle detection that allows to obtain the PSPACE upper bounds. This technique can be also used for $\mathcal{L}_{1}, \mathcal{L}_{2} \in\{K, T, B\}$. We took the decision to use $\mathcal{P} \mathcal{G} \mathcal{F}_{2}$ instead since it is an interesting fragment to equip with an analytic tableau-style calculus (see in [25] a labelled tableaux calculus for the modal logic $M L R_{2}$ of binary relations that corresponds roughly to WLGF2 augmented with other binary predicate symbols). Last but not least, the decision procedures we have defined could be straightforwardly (and more efficiently) reused in a tableaux calculus for $\left\langle\mathcal{L}_{1}, \mathcal{L}_{2}\right\rangle \in\{S 4, S 5\} \times\{T, B, S 4, S 5\}$. For instance, we can show that in a prefixed calculus one not need to consider prefixes of length greater than $\left(4 \times|\phi|^{2}+1\right)^{2}$. In a non-prefixed version, one does not need to apply the " $\pi$-rule" more than $\left(4 \times|\phi|^{2}+1\right)^{2}$ times on a branch to show that $\phi$ is valid. An analysis similar to the one in [8] about results in $[22,14]$ would be the right way to formally establish such results.

Besides, it is natural to extend the operator $\oplus \subseteq$ to an $n$-ary operator $n \geq 2$. Let $\left(\mathcal{C}_{i}\right)_{i \in\{1, \ldots, n\}}$ be $n \geq 2$ classes of monomodal frames. The class $\mathcal{C}_{1} \oplus \subseteq \ldots \oplus \subseteq \mathcal{C}_{n}$ of $n$-modal frames is defined as the class of frames $\left\langle W, R_{1}, \ldots, R_{n}\right\rangle$ such that for $i \in\{1, \ldots, n\},\left\langle W, R_{i}\right\rangle \in \mathcal{C}_{i}$ and $R_{1} \subseteq \ldots \subseteq R_{n}$. All the other notions can be naturally defined. One can show the following generalization:

Theorem 13. Let $\mathcal{L}_{1}, \ldots, \mathcal{L}_{n}$ be in $\{K, T, B, S 4, S 5\}, n \geq 2$. If there exist $i<$ $j \in\{1, \ldots, n\}$ such that $S A T\left(\mathcal{L}_{i} \oplus \subset \mathcal{L}_{j}\right)$ is EXPTIME-hard then $S A T\left(\mathcal{L}_{1} \oplus \subset\right.$ $\left.\ldots \oplus \subseteq \mathcal{L}_{n}\right)$ is EXPTIME-complete. Otherwise, if for $i \in\{1, \ldots, n\}, \mathcal{L}_{i}=S 5$, then $\bar{S} A T\left(\mathcal{L}_{1} \oplus \subseteq \ldots \oplus \subseteq \mathcal{L}_{n}\right)$ is $\mathrm{NP}$-complete otherwise $S A T\left(\mathcal{L}_{1} \oplus \subseteq \cdots \oplus \subseteq \mathcal{L}_{n}\right)$ is PSPACE-complete.

Acknowledgments. The author thanks the anonymous referees for useful remarks and suggestions. Special thanks are due to one of the referees for finding a mistake in the submitted version.

\section{References}

1. M. Baldoni. Normal Multimodal Logics: Automated Deduction and Logic Programming. $\mathrm{PhD}$ thesis, Università degli Studi di Torino, 1998.

2. B. Beckert and D. Gabbay. Fibring semantic tableaux. In H. de Swart, editor, TABLEAUX-8, pages 77-92. LNAI, Springer-Verlag, 1998.

3. J. van Benthem. Modal logic and classical logic. Bibliopolis, 1985.

4. J. van Benthem. Modal logic in two Gestalts. Technical report, ILLC, 1998.

5. P. Blackburn, M. de Rijke, and Y. Venema. Modal Logic. 2000. to appear.

6. M. Castilho, O. Gasquet, and A. Herzig. Formalizing action and change in modal logic I: the frame problem. J. of Logic and Computation, 1998. to appear.

7. M. Castilho and A. Herzig. An alternative to the iteration operator of propositional dynamic logic. Technical Report 96-05-R, IRIT, 1996.

8. S. Cerrito and M. Cialdea Mayer. A polynomial translation of S4 into T and contraction-free tableaux for S4. Logic Journal of the IGPL, 5(2):287-300, 1997.

9. A. Chagrov and M. Zakharyaschev. Modal Logic. Clarendon Press, Oxford, 1997.

10. C. Chen and I. Lin. The complexity of propositional modal theories and the complexity of consistency of propositional modal theories. In A. Nerode and Yu. V. Matiyasevich, editors, LFCS-3, St. Petersburg, pages 69-80. Springer-Verlag, LNCS $813,1994$. 
11. S. Demri. A class of decidable information logics. TCS, 195(1):33-60, 1998.

12. K. Fine and G. Schurz. Transfer theorems for multimodal logics. In Logic and Reality. Essays in Pure and Applied Logic. In Memory of Arthur Prior. OUP, 1992. To appear.

13. M. Fitting. Proof methods for modal and intuitionistic logics. D. Reidel Publishing Co., 1983.

14. M. Fitting. First-order modal tableaux. JAR, 4:191-213, 1988.

15. D. Gabbay. Fibred semantics and the weaving of logics Part 1: modal and intuitionistic logics. The Journal of Symbolic Logic, 61(4):1057-1120, 1996.

16. D. Gabbay and V. Shehtman. Products of modal logics, Part I. Logic Journal of the IGPL, 6(1):73-146, 1998.

17. R Goré. Tableaux methods for modal and temporal logics. In M. d'Agostino, D. Gabbay, R. Hähnle, and J. Posegga, editors, Handbook of Tableaux Methods, pages 297-396. Kluwer, 1999.

18. J. Halpern and Y. Moses. A guide to completeness and complexity for modal logics of knowledge and belief. Artificial Intelligence, 54:319-379, 1992.

19. E. Hemaspaandra. Complexity transfer for modal logic (extended abstract). In LICS-9, pages 164-173, 1994.

20. A. Heuerding. Sequent Calculi for Proof Search in Some Modal Logics. PhD thesis, University of Bern, 1998.

21. M. Kracht and F. Wolter. Simulation and transfer results in modal logic - A survey. Studia Logica, 59:149-1997, 1997.

22. R. Ladner. The computational complexity of provability in systems of modal propositional logic. SIAM Journal of Computing, 6(3):467-480, 1977.

23. M. Marx. Complexity of modal logics of relations. Technical report, ILLC, 1997. To appear in Annals of Pure and Applied Logic.

24. M. Marx. Complexity of products of modal logics. Journal of Logic and Computation, 9(2): 197-214, 1999.

25. M. Marx, S. Mikulás, and S. Schlobach. Tableau calculus for local cubic modal logic and its implementation. Logic Journal of the IGPL, 7(6):755-778, 1999.

26. F. Massacci. Single steps tableaux for modal logics. JAR, 1999. to appear.

27. Ch. Papadimitriou. Computational Complexity. Addison-Wesley Publishing Company, 1994.

28. V. Pratt. Models of program logics. In Proceedings of the 20th IEEE Symposium on Foundations of Computer Science, pages 115-122, 1979.

29. A. Sistla and E. Clarke. The complexity of propositional linear temporal logic. JACM, 32(3):733-749, 1985.

30. E. Spaan. Complexity of Modal Logics. PhD thesis, ILLC, 1993.

31. E. Spaan. The complexity of propositional tense logics. In M. de Rijke, editor, Diamonds and Defaults, pages 287-309. Kluwer, 1993.

32. M. Tiomkin and M. Kaminsky. Nonmonotonic default modal logics. JACM, 38:963-984, 1991.

33. D. Vakarelov. Modal characterization of the classes of finite and infinite quasiordered sets. In P. Petkov, editor, Mathematical Logic, pages 373-387. Plenum Press, New-York, 1990.

34. D. Vakarelov. Modal logics for knowledge representation systems. TCS, 90:433456, 1991.

35. L. Viganò. Labelled Non-Classical Logics. Kluwer, 2000.

36. A. Visser. A course on bimodal provability logic. Annals of Pure and Applied Logic, 73:109-142, 1995. 